\title{
Evaluation of left renal vein and inferior vena cava variations through routine abdominal multi-slice computed tomography
}

\author{
N. Boyaci, E. Karakas, D.S. Dokumacı, S. Yildiz, H. Cece \\ Department of Radiology, Medical School, Harran University, Sanliurfa, Turkey \\ [Received 24 August 2013; Accepted 17 December 2013]
}

\begin{abstract}
Background: The aim of this study was to evaluate the frequency of left renal vein (LRV) and inferior vena cava (IVC) variations and the effect of gender on this frequency, as well as the presence of associated abdominal pathologies.

Materials and methods: Multi-slice computed tomography (MSCT) images from 746 patients were evaluated retrospectively.

Results: Left renal vein variations were identified in $9.8 \%$ of cases, while retroaortic $L R V$ (RLRV) and circumaortic LRV (CLRV) were found in $7.4 \%$ and $2.4 \%$ of cases, respectively. No significant correlation was found between gender and $L R V$ variations $(p=0.797)$. Of the cases with $L R V$ and IVC variations, $73 \%$ had abdominal pathologies, the most common of which were nephrolithiasis, which appeared in 18 (32\%) cases, and renal cysts, which appeared in 14 (25.4\%) cases. Conclusions: MSCT is a rapid and reliable method of identifying LRV and IVC variations and associated abdominal pathologies. (Folia Morphol 2014; 73, 2: 159-163)
\end{abstract}

Key words: inferior vena cava, left renal veins, multi-slice computed tomography

\section{INTRODUCTION}

Left renal vein (LRV) and inferior vena cava (IVC) variations are caused by variations in the anastomoses of the posterior cardinal, subcardinal, and supracardinal veins during embryonic development and can take a number of forms [2]. LRV variations include retroaortic LRV (RLRV) and circumaortic LRV (CLRV), which can be found in $2.1 \%$ and $8.7 \%$ of cases, respectively [10]. Inferior vena cava variations include double IVC, left IVC, and azygos continuation of the IVC, which occur in $0.2-3 \%, 0.2-0.5 \%$, and $0.6 \%$ of cases, respectively $[6,10]$. It is crucial to determine the presence of LRV and IVC variations before beginning retroperitoneal surgical interventions, interventional vascular procedures, or donor nephrectomies for transplantation, and the vascular structures and associated abdominal pathologies can be evaluated quickly and reliably by means of multi-slice computed tomography (MSCT) [8]. The purpose of this study is to evaluate the frequency of LRV and IVC variations and the effect of gender on this frequency, as well as the presence of associated abdominal pathologies.

\section{MATERIALS AND METHODS}

\section{Patients and study design}

The study included 395 male and 351 female patients with a mean age \pm standard deviation of $40.69 \pm 23$ (range 1-95) years. Of the 746 total cases, 570 were 18 years old or older. The presence of $\mathrm{LRV}$ and IVC variations was evaluated retrospectively during routine abdominal MSCT examinations of the

Address for correspondence: N. Boyaci, MD, Department of Radiology, Medical School, Harran University, 63300, Sanliurfa, Turkey, tel: +90 (414) 31822 88, fax: +90 (414) 31831 92, e-mail: drnurefsan@yahoo.com 
Table 1. Distribution of patients by gender

\begin{tabular}{lccc}
\hline & Female & Male & P \\
\hline Frequency & $351(47.1 \%)$ & $395(52.9 \%)$ & \\
Age $($ mean \pm SD) & $41.24 \pm 22.96$ & $40.20 \pm 23.55$ & 0.543 \\
\hline
\end{tabular}

patients that were carried out because of abdominal problems between January and July 2012. Patients with nephrectomy and some of the paediatric patients were excluded from the study because their vascular structures could not be evaluated, due to motion artefact and poor opacification. Approval was obtained from the local ethics committee prior to the study. The LRV was evaluated via axial computerised tomography (CT) images from the renal hilum to the IVC and was considered structurally normal when it drained into the IVC by crossing anterior to the aorta. The LRV was considered RLRV when it drained into the IVC by passing posterior to the aorta and was considered CLRV when one LRV crossed anterior to the aorta and another crossed posterior to the aorta to join the IVC. The presence and localisation of the IVC were evaluated via axial CT images and were considered structurally normal when on the right side of the aorta. When the IVC was on the left side of the aorta, this was defined as a transposed left IVC, and when IVCs were found on both sides of the aorta, this was reported as a double IVC. A prerenal IVC was defined as an azygos continuation of the IVC that passes posterior to the diaphragmatic crura to enter the thorax as the azygos vein.

\section{Imaging method}

Abdominal CT examinations were performed using a multi-detector row CT (16-slice, Siemens, Somatom Emotion, Germany) and following standard protocols. Slices were obtained from the region extending from the dome of the diaphragm to the end of the pelvis. Examinations were performed using intravenous contrast medium during the portal venous phase. A non-ionic contrast medium with an iodine concentration of $300 \mathrm{mg} / \mathrm{cc}$ was administered intravenously into the antecubital vein with an automatic injector pump at a dose of $1-2 \mathrm{mg} / \mathrm{kg}$ after a $70 \mathrm{~s}$ delay time. For the examinations requiring intestinal opacification, a non-ionic contrast medium with iodine diluted in water was orally administered to the patients before the examination, in addition to intravenous contrast medium.
The obtained axial images were uploaded onto a Picture Archiving Communication System (PACS) workstation, and the examination results were evaluated together with the axial images and reformatted multi-planar reconstruction (MPR) images as necessary. Image analysis was performed by the same radiologist in all cases.

\section{Statistical analysis}

The data obtained were processed using the Statistical Package for the Social Sciences (SPSS) Version 15.0 (SPSS Inc, Chicago, IL, USA), and the statistical analysis was evaluated at a $p<0.05$ significance level. An independent t-test was used to compare the data for males and females. The relationships between the categorical variables were determined using a $\chi^{2}$ test. The percentages of detectable LRV variations and IVC variations were calculated.

\section{RESULTS}

A total of 746 patients were enrolled in the study, 395 (52.9\%) males and 351 (47.1\%) females (Table 1). LRV and IVC variations were found in 75 (10.1\%) cases, while LRV variations were identified in $73(9.8 \%)$ cases, of which 55 (7.4\%) were RLRV (Fig. 1) and 18 (2.4\%) were CLRV (Fig. 2). Among the female patients, total LRV variations, RLRVs, and CLRVs numbered 36 (10.3\%), 28 (8\%), and 8 (2.3\%), respectively, and the corresponding values among men were 37 (9.3\%), 27 (6.8\%), and 10 (2.5\%). No statistically significant correlation was found between gender and overall LRV variations, RLRV, or CLRV ( $p=0.801, p=0.551, p=0.823$, respectively). Only $2(0.3 \%)$ patients had azygos continuations of the IVC (Fig. 3). No other variations of the IVC were observed.

Overall, 1 male and 1 female patient had IVC variations, with no statistically significant correlation being found between gender and the presence of variations $(p=0.933)$. The numbers and percentages of LRV and IVC variations among the patients are presented in Table 2.

During the CT, 55 (73.3\%) cases with vascular variations were found to have various abdominal pathologies, of which 18 (32\%) had nephrolithiasis, 


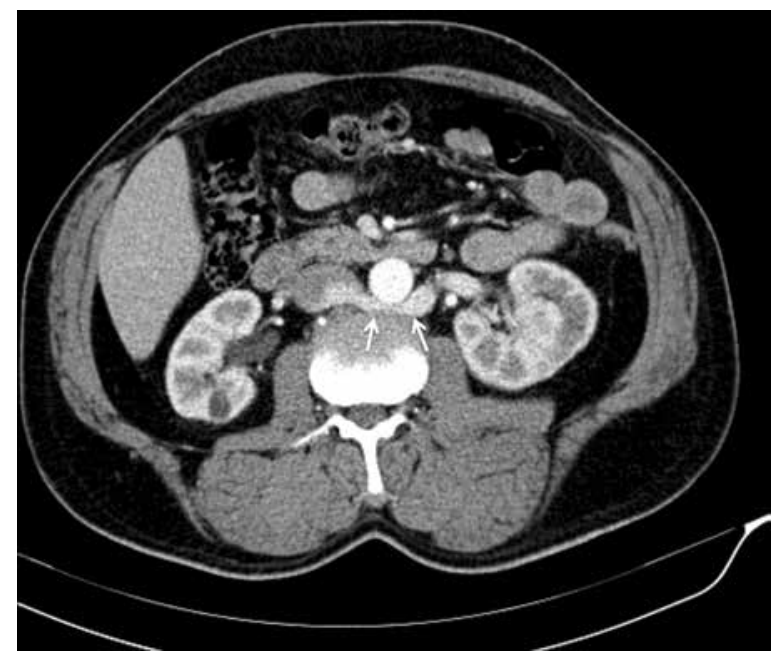

Figure 1. Axial computed tomography image of a 55-year-old male patient demonstrates retroaortic left renal vein (arrows).

$14(25.4 \%)$ had renal cysts, and $8(14.5 \%)$ had hepatic hydatid cysts. Other $\mathrm{CT}$ findings included hepatic and splenic lacerations, ascites, ovarian cysts, uterine myomas, perforated appendicitis, and ileus.

\section{DISCUSSION}

In a retrospective study of 746 patients, LRV and IVC variations were found in a total of $75(10.1 \%)$ cases: $73(9.7 \%)$ had LRV variations and $2(0.3 \%)$ had IVC variations. No significant correlation was found between gender and variations in the LRV or IVC. A further $73.3 \%$ of cases with LRV variations were found to have abdominal pathologies on the $\mathrm{CT}$, the most common of which were nephrolithiasis, in 18 (32\%) cases, and renal cysts, in $14(25.4 \%)$ cases.

There have been several studies investigating the frequency of LRV and IVC variations using enhancement helical CT, MSCT angiography, conventional angiography, and magnetic resonance angiography. These studies have reported a variation frequency ranging from $3 \%$ to $10 \%$ for $\operatorname{LRV}[1,3-5,12,15]$. According to our study, variations of the LRV or IVC exist in $10.1 \%$ of society. Therefore, LRV or IVC vascular variations exist in one out of every 10 people. Imaging the renal vascular anatomy preoperatively is essential in invasive therapy planning. Vascular variations should be known before surgery because this will prevent vascular complications. The presence of LRV and IVC variations should be investigated prior to retroperitoneal surgical interventions, partial nephrectomies, laparoscopic procedures, and donor nephrectomies for transplantation [13]. In the present
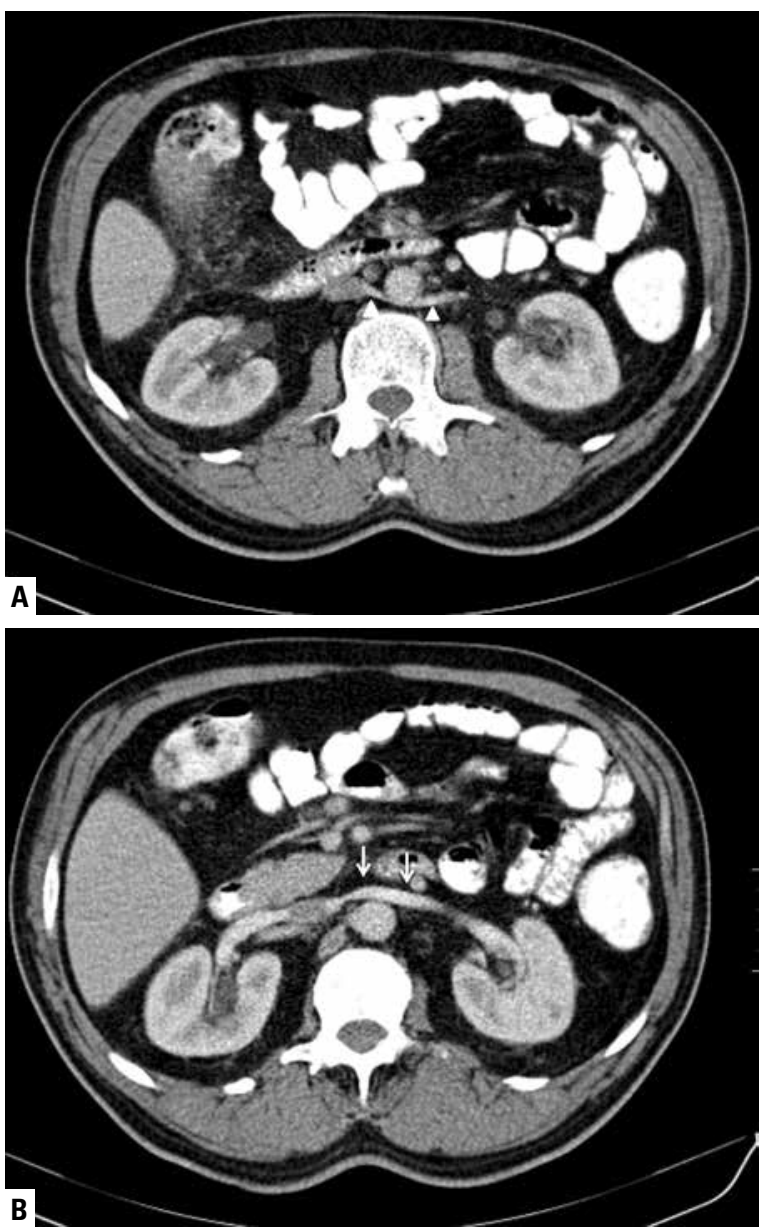

Figure 2. Axial computed tomography images of a 55-year-old male patient demonstrate circumaortic left renal vein with both $(\mathbf{A})$ retroaortic (arrowheads) and (B) preaortic (arrows) components.

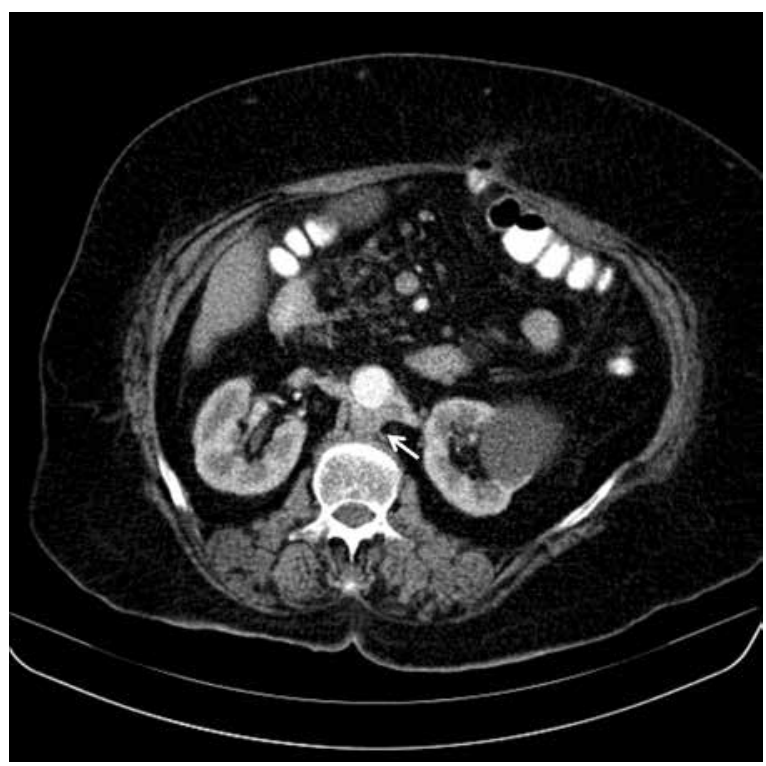

Figure 3. Axial computed tomography images of a 73-year-old female patient demonstrate azygos continuation of the inferior vena cava (arrow). 
Table 2. Distribution of left renal vein (LRV) and inferior vena cava (IVC) variations in patients

\begin{tabular}{lccc}
\hline & Male & Female & Total \\
\hline LRV variations: & & & $55(7.4 \%)$ \\
Retroaortic LRV & $27(6.8 \%)$ & $28(8 \%)$ & $18(2.4 \%)$ \\
Circumaortic LRV & $10(2.5 \%)$ & $8(2.3 \%)$ & \\
IVC variations: & & & $2(0.3 \%)$ \\
Azygos & $1(0.3 \%)$ & $1(0.3 \%)$ & $75(10.1 \%)$ \\
Total & $38(9.6 \%)$ & $37(10.5 \%)$ & \\
\hline
\end{tabular}

study, the RLRV rate was $6.9 \%$, which is quite high when compared with those of other studies [1, 3, $4,12,15]$. As in many other studies, no significant correlation was found between LRV variations and gender in our study $[5,7,15]$.

The development of LRV and IVC occurs between the $4^{\text {th }}$ and $8^{\text {th }}$ gestational weeks as a result of a number of anastomoses of the posterior cardinal, subcardinal, and supracardinal veins [12]. Many variations may develop during this complex formation process due to changes that may occur during various stages [2]. A transposed left IVC runs parallel to the aorta on its left side, and this condition has a prevalence of $0.2-0.5 \%$ [10]. A double IVC refers to the presence of the IVC on the left side as well, and the prevalence of this condition has been reported to be $0.2-3.0 \%$ [10]. In an azygos continuation of the IVC, the IVC merges with the azygos vein by passing posterior to the diaphragmatic crura in the prerenal segment. Its prevalence was reported to be $0.6 \%$ [6]. In our study, this variation was found in $2(0.3 \%)$ cases. In 1 case of azygos continuation of the IVC, the patient also had situs ambiguous-heterotaxy syndrome [9]. It is vital to be aware of the presence of a LRV and IVC variation prior to surgical interventions or the implantation of vena cava filters because this may prevent possible haemorrhages and potentially fatal complications. Furthermore, the recognition of such vascular structures, which may be confused with lymphadenopathies, is crucial for disease staging and management planning.

IVC and LRV variations can be evaluated via venography, which is an invasive interventional method [11] or evaluated through colour Doppler ultrasonography (CDUS), which is a non-invasive imaging technique. However, CDUS is operator-dependent, and it can be difficult to evaluate obese or immobile patients. Venous structures can be evaluated via a magnetic resonance imaging, without the use of contrast media [5], although magnetic resonance imaging is both time-consuming and expensive when compared with CT. The disadvantages of CT are its use of ionizing radiation and the possible nephrotoxicity of the contrast media.

In our study, the most common abdominal pathologies in cases with vascular variation were nephrolithiasis, in 18 (32\%) cases, and renal cysts, in 14 (25.4\%) cases. This may be due to the negative effects of these variations on renal function. In a previous study, an increase in the intrarenal venous impedance index was found to result from the compression of the retroaortic LRV behind the aorta [14]. Such variations may have negative effects on renal haemodynamics.

Our study has several limitations. Principally, all cases included in the study were patients who had undergone abdominal CT examinations for various reasons, and because healthy people cannot be given $\mathrm{CT}$ scans due to the radiation risk, the present study is unable to make a comparison with the variation rate in healthy people. The main strength of the present study is that it includes the large study population examined via MSCT.

\section{CONCLUSIONS}

In conclusion, MSCT is a rapid and reliable method of identifying LRV and IVC variations and associated abdominal pathologies. The presence of the LRV and IVC variations is not related to gender. Due to the contribution of the MPR-reformatted images, a reliable assessment can be made via MSCT. Incidentally, detected vascular variations should always be reported when found during routine CT scans. Renal pathologies are the most common pathologies seen in cases with renal vein variations. Further studies of larger series are needed to investigate the effects of these variations on renal haemodynamics and function. 


\section{REFERENCES}

1. Atalar MH, Kosar MI, Salk I, Isleyen M (2012) Left renal vein abnormalities detected during routine abdominal computed tomography imaging: clinico-radiological significance. Folia Morphol, 71: 168-172.

2. Bass JE, Redwine MD, Kramer LA, Huynh PT, Harris JH, Jr. (2000) Spectrum of congenital anomalies of the inferior vena cava: cross-sectional imaging findings. Radiographics, 20: 639-652.

3. Bouali O, Labarre D, Molinier F, Lopez R, Benouaich V, Lauwers F, Moscovici J (2012) Anatomic variations of the renal vessels: focus on the precaval right renal artery. Surg Radiol Anat, 34: 441-446.

4. Dilli A, Ayaz UY, Kaplanoglu H, Saltas H, Hekimoglu B (2013) Evaluation of the left renal vein variations and inferior vena cava variations by means of helical computed tomography. Clin Imag, 37: 530-535.

5. Dilli A, Ayaz UY, Karabacak OR, Tatar IG, Hekimoglu B (2012) Study of the left renal variations by means of magnetic resonance imaging. Surg Radiol Anat, 34: 267-270.

6. Ginaldi S, Chuang VP, Wallace S (1980) Absence of hepatic segment of the inferior vena cava with azygous continuation. J Comput Assist Tomogr, 4: 112-114.

7. Koc Z, Ulusan S, Oguzkurt L, Tokmak N (2007) Venous variants and anomalies on routine abdominal multi-detector row CT. Eur J Radiol, 61: 267-278.

8. Kumar S, Neyaz Z, Gupta A (2010) The utility of 64 channel multidetector CT angiography for evaluating the renal vascular anatomy and possible variations: a pictorial essay. Korean J Radiol, 11: 346-354.

9. Minniti S, Visentini S, Procacci C (2002) Congenital anomalies of the venae cavae: embryological origin, imaging features and report of three new variants. Eur Radiol, 12: 2040-2055.

10. Phillips E (1969) Embryology and anomalies. In: Ferris EJ, Hipona FA, Kahn PC, Phillips E, Shapiro JH eds. Venography of the inferior vena cava and its branches. Williams \& Wilkins, Baltimore, pp. 1-32.

11. Satyapal KS, Kalideen JM, Haffejee AA, Singh B, Robbs JV (1999) Left renal vein variations. Surg Radiol Anat, 21: 77-81.

12. Trigaux JP, Vandroogenbroek S, De Wispelaere JF, Lacrosse M, Jamart J (1998) Congenital anomalies of the inferior vena cava and left renal vein: evaluation with spiral CT. J Vasc Interv Radiol, 9: 339-345.

13. Turkvatan A, Ozdemir M, Cumhur T, Olcer T (2009) Multidetector $\mathrm{CT}$ angiography of renal vasculature: normal anatomy and variants. Eur Radiol, 19: 236-244.

14. Yagci B, Tavasli B, Karabulut N, Kiroglu Y (2008) Clinical significance and renal haemodynamics of incidentally detected retroaortic left renal vein: assessment with venous Doppler sonography. Br J Radiol, 81: 187-191.

15. Yesildag A, Adanir E, Koroglu M, Baykal B, Oyar O, Gulsoy UK (2004) [Incidence of left renal vein anomalies in routine abdominal CT scans]. Tani Girisim Radyol, 10: 140-143. 